\title{
20(S)-Rg3 blocked epithelial-mesenchymal transition through DNMT3A/miR-145/FSCN1 in ovarian cancer
}

\author{
Jie $\mathrm{Li}^{1,2,3}$, Jiaojiao Lu ${ }^{1,2}$, Zhongxue $\mathrm{Ye}^{4}$, Xi Han ${ }^{1,2}$, Xia Zheng ${ }^{1,2}$, Huilian Hou ${ }^{5}$, Wei \\ Chen ${ }^{6}, \mathrm{Xu} \mathrm{Li}^{1,2}$ and Le Zhao ${ }^{1,2}$ \\ ${ }^{1}$ Center for Translational Medicine, The First Affiliated Hospital of Xi'an Jiaotong University, Xi'an, China \\ ${ }^{2}$ Key Laboratory for Tumor Precision Medicine of Shaanxi Province, The First Affiliated Hospital of Xi'an Jiaotong University, \\ Xi'an, China \\ ${ }^{3}$ Department of Gynecology and Obstetrics, The First Affiliated Hospital of Xi'an Jiaotong University, Xi'an, China \\ ${ }^{4}$ Department of Gynecology, Ningbo No. 2 Hospital, Ningbo, China \\ ${ }^{5}$ Department of Pathology, The First Affiliated Hospital of Xi'an Jiaotong University, Xi'an, China \\ ${ }^{6}$ Center for Laboratory Medicine, The First Affiliated Hospital of Xi'an Jiaotong University, Xi'an, China \\ Correspondence to: Le Zhao, email: zhaole2@mail.xjtu.edu.cn \\ Xu Li, email: lixu56@mail.xjtu.edu.cn \\ Keywords: ovarian cancer, ginsenoside, microRNA, methylation, epithelial-mesenchymal transition \\ Received: August 20, $2016 \quad$ Accepted: May 29, $2017 \quad$ Published: June 15, 2017 \\ Copyright: Li et al. This is an open-access article distributed under the terms of the Creative Commons Attribution License 3.0 \\ (CC BY 3.0), which permits unrestricted use, distribution, and reproduction in any medium, provided the original author and source \\ are credited.
}

\section{ABSTRACT}

Epithelial-mesenchymal transition (EMT) is one of the key mechanisms mediating cancer progression. MicroRNAs (miRs) are essential regulators of gene expression by suppressing translation or causing degradation of target mRNA. Growing evidence illustrates the crucial roles of miRs dysregulation in cancer development and progression. Here, we have found for the first time that the ginsenoside 20 (S)Rg3, a pharmacologically active component of Panax ginseng, potently increases miR-145 expression by downregulating methyltransferase DNMT3A to attenuate the hypermethylation of the promoter region in the miR-145 precursor gene. Restoration of DNMT3A reverses the inhibitory effect of $20(S)-R g 3$ on EMT. FSCN1 is verified as the target of miR-145 to suppress EMT in human ovarian cancer cells. The results from nude mouse xenograft models further demonstrate the suppressive effect of miR-145 on malignant progression of ovarian cancer. Taken together, our results show that 20(S)-Rg3 blocks EMT by targeting DNMT3A/miR-145/FSCN1 pathway in ovarian cancer cells, highlighting the potentiality of $20(\mathrm{~S})-\mathrm{Rg} 3$ to be used as a therapeutic agent for ovarian cancer.

\section{INTRODUCTION}

Ovarian cancer is the most lethal gynecological tumor, existing predominantly in the form of epithelial ovarian cancer $(\mathrm{EOC})[1,2]$. The prognosis of EOC patients remains poor, and cancer metastasis and recurrence are the major causes of death. Emerging evidence suggests that the epithelial-mesenchymal transition (EMT), the conversation of epithelial cells to fibroblast-like cells mainly characterized by loss of epithelial molecules, acquisition of mesenchymal markers, enhancement of cell mobility and invasion, plays a crucial role in the progression of EOC by increasing cancer cell invasion and metastasis [3]. Thus, targeting EMT process for novel anticancer drug discovery is highly significant for the clinical benefits of ovarian cancer patients.

Ginsenosides are the pharmacologically active components of Panax ginseng that has long been utilized as a traditional Chinese medicine for officinal or recuperative purposes [4-6]. To date, more than 100 ginsenoside compounds have been identified [7], among which Rg3 is one of the bioactive extracts with anti-tumor effect $[8,9]$. Ginsenoside $\operatorname{Rg} 3$ has two stereoisomers 20(R)-Rg3 and 20(S)-Rg3, which differ in the orientation 
of the hydroxyl $(\mathrm{OH})$ group on carbon-20 [5]. We have found that $20(\mathrm{~S})-\mathrm{Rg} 3$, rather than $20(\mathrm{R})-\mathrm{Rg} 3$, has robustly blocked hypoxia-induced EMT in ovarian cancer.

Many EMT players such as E-cadherin transcription repressors including snail, zeb and Twist, and microRNAs have been identified so far. MicroRNAs are small noncoding RNAs that modulate gene expression at the posttranscriptional level through complementary binding to 3'UTR of target mRNAs [10]. Some microRNAs including miR-145, miR-200, and miR-29b, to name a few, have been suggested as EMT repressors in various types of cancer [11-14]. miR-145 has been downregulated in many cancers [15-19], functioning as tumor suppressor to inhibit tumor cell growth and survival, induce cell apoptosis and cell cycle arrest, and attenuate tumor cell migration and invasion via targeting various molecules [16-18, 20-23]. Reduction of miR level in cancers can be associated with aberrant epigenetic regulation such as DNA hypermethylation [24]. DNMT3A and DNMT3B are two major de novo DNA methyltransferases in mammals, while DNMT1 is in charge of maintenance of DNA methylation [25]. DNMT3A and DNMT3B have been reported upregulated in ovarian cancers [26]. Both of them have been able to hypermethylate the promoter region in microRNA precursor genes and thus inversely regulate microRNA transcription.

In the present study, we discovered that 20(S)Rg3 enhanced miR-145 expression by downmodulating DNMT3A to attenuate the methylation level in the promoter region of miR-145 precursor gene. The promotion of miR145 by $20(\mathrm{~S})-\mathrm{Rg} 3$ directly targeted FSCN1 to reverse EMT in vitro and vivo. These results not only uncovered the novel anti-cancer mechanism of $20(\mathrm{~S})-\mathrm{Rg} 3$, but also revealed the regulatory pathway for miR-145 expression.

\section{RESULTS}

\section{0(S)-Rg3 reversed EMT via upregulating miR-145}

In 20(S)-Rg3-treated SKOV3 and 3AO cells, epithelial marker E-cadherin was up-regulated, while mesenchymal markers $\mathrm{N}$-cadherin and vimentin were down-regulated (Figure 1A), indicating that 20(S)-Rg3 reversed EMT in SKOV3 and 3AO ovarian cancer cells. Meanwhile, 20(S)-Rg3 caused E-cadherin increase and $\mathrm{N}$-cadherin and vimentin decrease in A2780 ovarian cancer cells and HEC-1-B endometrial cancer cells (Supplementary Figure 1A). Since miR-145 was reported downregulated in ovarian cancer cells and involved in EMT in many types of cancer, we explored the effect of $20(\mathrm{~S})-\mathrm{Rg} 3$ on miR145. In both SKOV3 and 3AO cells, 20(S)-Rg3 stimulated miR-145 expression (Figure 1B). Transfection of miR-145 inhibitor (Supplementary Figure 2) into 20(S)-Rg3-treated cells (Figure 1C) reversed 20(S)-Rg3-rendered E-cadherin upregulation and $\mathrm{N}$-cadherin and vimentin downregulation (Figure 1D, Supplementary Figure 3). Inhibition of miR-
145 also blocked the inhibitory effect of $20(\mathrm{~S})-\mathrm{Rg} 3$ on the migration and invasion of both SKOV3 and $3 \mathrm{AO}$ cells (Figure 1E). We next overexpressed miR-145 to examine its effect on EMT of ovarian cancer cells. miR145 overexpression (Figure $1 \mathrm{~F}$ ) increased E-cadherin while decreased N-cadherin and vimentin (Figure 1G). Meanwhile, overexpression of miR-145 largely inhibited migration and invasion of both SKOV3 and 3AO cells (Figure 1H). These results showed that $20(\mathrm{~S})-\mathrm{Rg} 3$ upregulated miR-145 to reverse EMT in ovarian cancer cells.

\section{0(S)-Rg3 upregulated miR-145 via suppressing DNMT3A to demethylate pre-miR-145 gene}

Since DNA hypermethylation has been connected to miR-145 deregulation in prostate cancer [27], we compared the methylation status of miR-145 precursor gene in 20(S)-Rg3-treated cells relative to non-treated cells. The initial assessment of potential $\mathrm{CpG}$ islands in the 2000 bp upstream genomic sequence encoding premiR-145 found no $\mathrm{CpG}$ islands but some $\mathrm{CG}$ sites. The MSP results illustrated that the methylation level in the promoter region of pre-miR-145 promoter was decreased in 20(S)-Rg3-treated SKOV3 and 3AO cells (Figure $2 \mathrm{~A})$. We thus detected the influence of $20(\mathrm{~S})-\mathrm{Rg} 3$ on the expression of DNMT family members. Western blot results showed that DNMT3A, the DNA methyltransferase responsible for de novo methylation, was obviously decreased while DNMT1 and DNMT3B were remained unchanged in 20(S)-Rg3-treated SKOV3 and 3AO cells (Figure 2B). DNMT3A plasmid was transfected into 20(S)-Rg3-treated cells to restore the expression of DNMT3A (Figure 2C), which reversed the 20(S)-Rg3triggered upregulation of miR-145 (Figure 2D). In parallel, MSP results showed that overexpression of DNMT3A increased the methylation level in the promoter region of pre-miR-145 in 20(S)-Rg3-treated SKOV3 and 3AO cells (Figure 2E). These results indicated that 20(S)-Rg3 enhanced miR-145 via inhibiting DNMT3A expression and thus relieved the methylation restrain on pre-miR-145 transcription.

We next assessed the effects of DNMT3A on the anti-EMT activity of 20(S)-Rg3. DNMT3A overexpression reversed 20(S)-Rg3-triggered E-cadherin upregulation, $\mathrm{N}$-cadherin and vimentin downregulation (Figure $2 \mathrm{~F}$ ). And the attenuation of migration and invasion of 20(S)-Rg3treated cells was largely reversed by ectopic expression of DNMT3A (Figure 2G). These results showed that 20(S)Rg3 blocked EMT through downregulating DNMT3A in ovarian cancer cells.

\section{miR-145 inhibited DNMT3A-promoted EMT}

We further studied the role of miR-145 in DNMT3A-promoted EMT. Ectopic expression of miR-145 in DNMT3A-overexpressed cells (Figure 3A) did not in turn affect DNMT3A expression (Figure 3B), but blunted 
the EMT-inducible activity of DNMT3A as shown by restoration of E-cadherin, loss of $\mathrm{N}$-cadherin and vimentin (Figure 3C), and attenuation of cell motility and invasion (Figure 3D). These results showed that DNMT3A induced EMT via inhibiting miR-145.

\section{miR-145 directly targeted FSCNI to inhibit EMT}

Next we explored the target of miR-145 to block EMT. We first predicted putative target genes of miR145 by searching the TargetScan database (release 5.1, http://www.targetscan.org/), and FSCN1 was chosen to be experimentally verified. 3'-UTRs of FSCN1 containing the wild-type or mutant putative miR-145 binding site were cloned into a luciferase reporter plasmid, respectively. Specifically, although 4 conserved miR145 seeding sequences were predicted to exist in the 3 '-
UTR of FSCN1, only one of them was experimentally confirmed as an actual binding site which was cloned into a luciferase reporter plasmid in our study [28]. The luciferase reporter assay showed that luciferase activity was significantly inhibited in cells co-transfected with miR-145 mimic and FSCN1 WT-3' UTR vector, while no changes of luciferase activity were detected in cells transfected with miR-145 mimic and luciferase reporter plasmids containing the mutant seeding sequence (Figure 4A). Additionally, western blot analysis showed that miR145 overexpression diminished FSCN1 expression level (Figure 4B). These data indicated the direct suppressive effect of miR-145 on FSCN1. Since the role of FSCN1 in EMT was not fully explored, we investigated whether FSCN1 mediated the inhibitory effect of miR-145 on EMT. In cells overexpressed both FSCN1 and miR145 , the anti-EMT effect of miR-145 as evidenced by
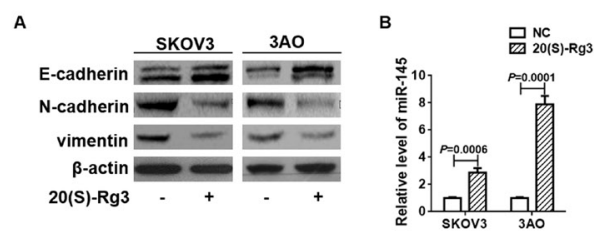

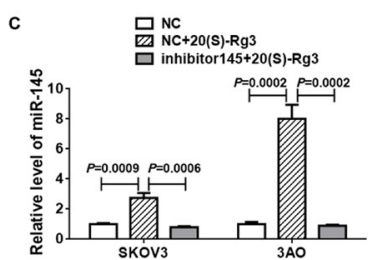

E

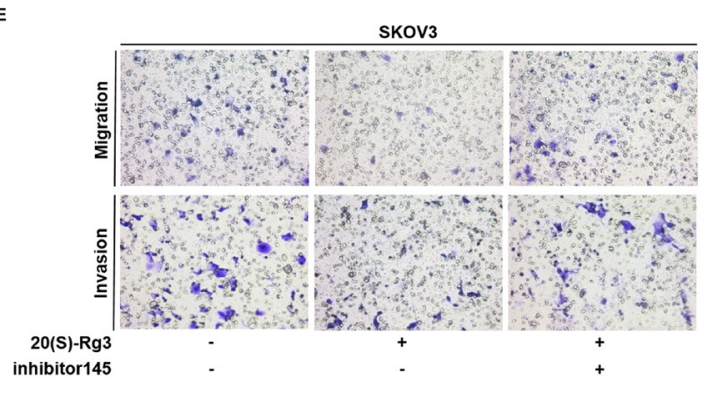

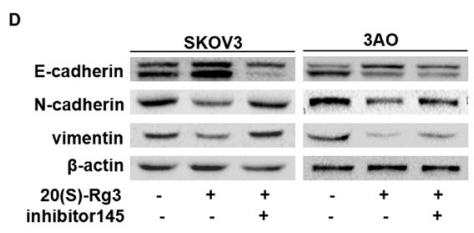

inhibitor145

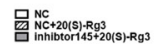
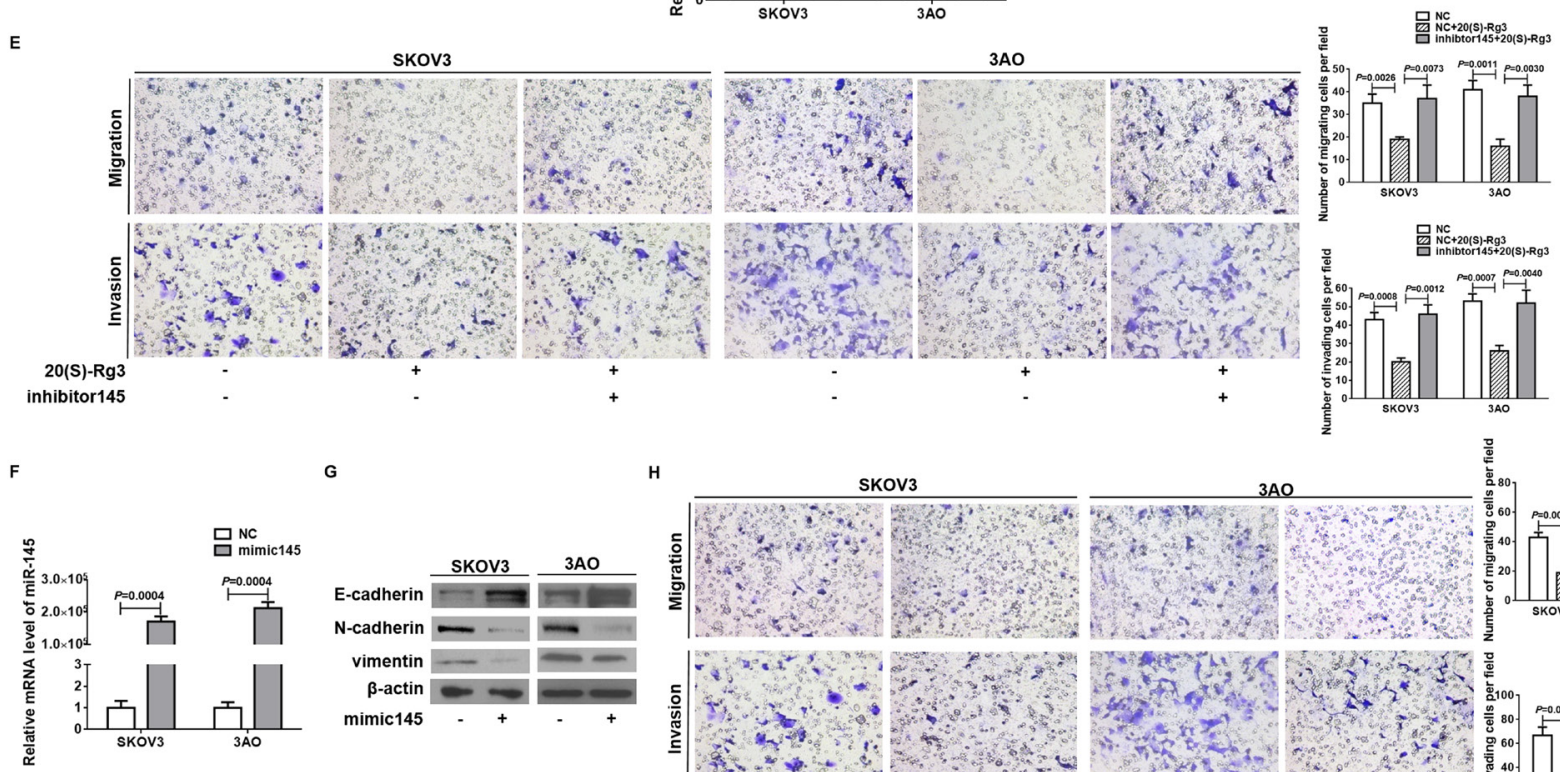

H
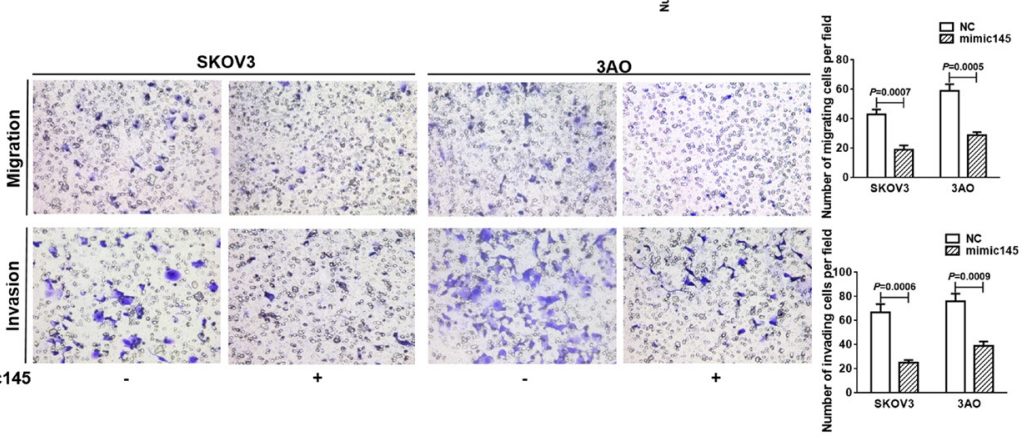

Figure 1: 20(S)-Rg3 reversed EMT via upregulating miR-145. (A) Western blot analysis showed that epithelial marker E-cadherin was up-regulated while mesenchymal markers N-cadherin and vimentin were down-regulated at protein level in cells treated by $20(\mathrm{~S})$-Rg3. (B) qRT-PCR showed that 20(S)-Rg3 increased miR-145 level in SKOV3 and 3AO cells. (C) qRT-PCR showed that inhibition of miR145 antagonized 20(S)-Rg3-triggered increase of miR-145. (D) Western blot analysis indicated that E-cadherin increase, N-cadherin and vimentin decrease caused by 20 (S)-Rg3 were reversed by concomitant miR-145 downregulation. (E) In vitro migration and invasion assay showed that transfection of miR-145 inhibitor reversed the inhibitory effect of 20(S)-Rg3 on cell motility and invasion (200×). (F) qRTPCR showed that transfection of miR-145 mimic significantly increased miR-145 level in SKOV3 and 3AO cells. (G) Western blot assays showed that the expression of E-cadherin was increased and N-cadherin and vimentin were decreased in SKOV3 and 3AO cells transfected with mimic145. (H) In vitro migration and invasion assays showed that overexpression of miR-145 in SKOV3 and $3 \mathrm{AO}$ cells reduced the cell invading capacity $(200 \times)$. All experiments were carried out in triplicate and the results were presented as means \pm SE. t-test. 
A

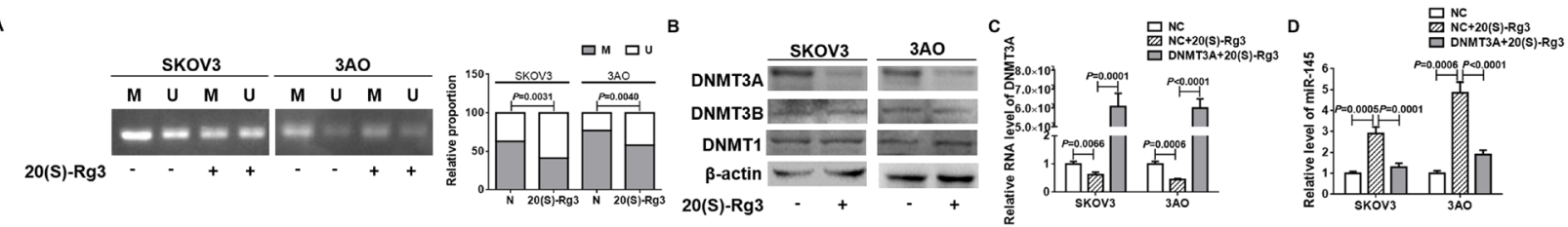

E
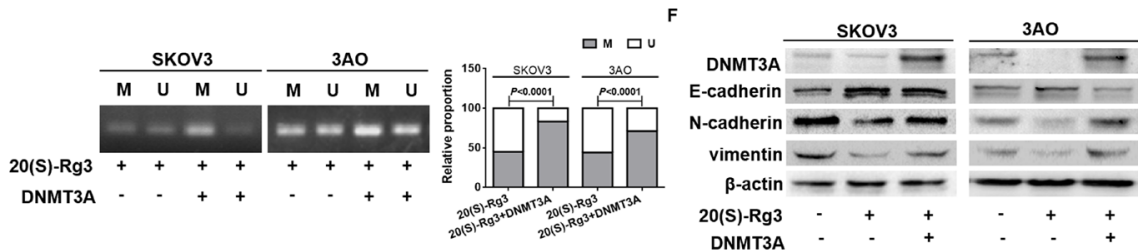

G
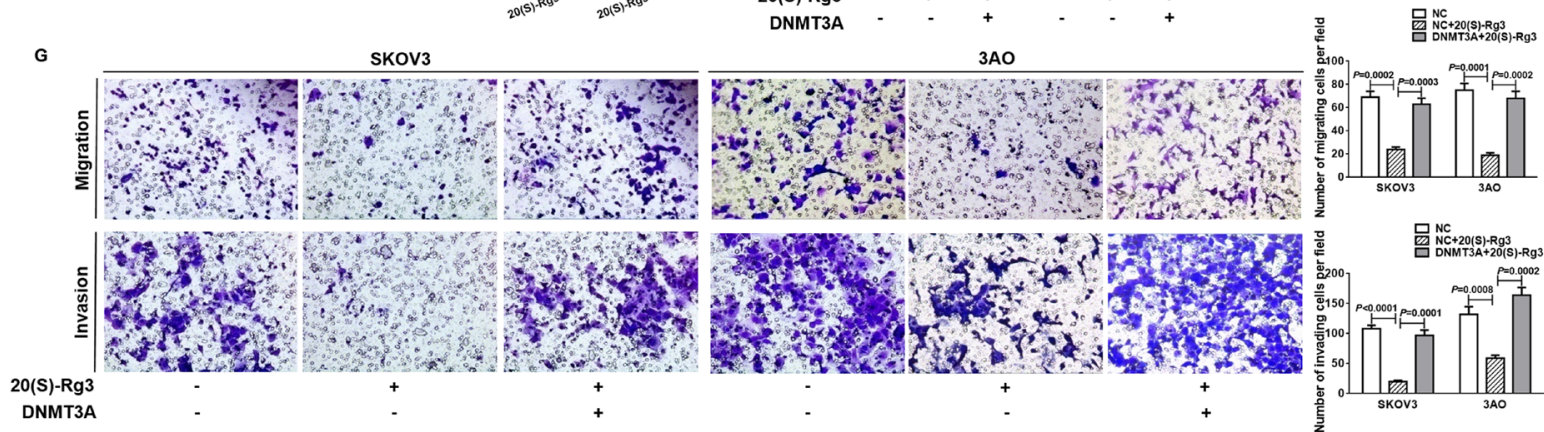

Figure 2: 20(S)-Rg3 upregulated miR-145 via suppressing DNMT3A-mediated methylation of pre-miR-145. (A) MSP showed that the methylation level in the pre-miR-145 promoter of SKOV3 and 3AO cells was decreased by 20(S)-Rg3. M: methylated product, U: unmethylated product. Fisher exact test. (B) Western blot analysis indicated that DNMT3A rather than DNMT3B and DNMT1 was significantly decreased in SKOV3 and 3AO cells treated by 20(S)-Rg3. (C) qRT-PCR showed that transfection of DNMT3A plasmid rescued DNMT3A level in 20(S)-Rg3-treated cells. $t$ test. (D) qRT-PCR showed overexpression of DNMT3A reversed 20(S)-Rg3-induced miR-145. (E) MSP showed the methylation level in the pre-miR-145 promoter was increased in cells treated with both 20(S)-Rg3 and DNMT3A plasmid compared to that in cells treated with 20(S)-Rg3 alone. M: methylated product, U: unmethylated product. Fisher exact test. (F) Western blot analysis showed that restoration of DNMT3A reversed 20(S)-Rg3-induced E-cadherin increase, N-cadherin and vimentin decrease. (G) In vitro migration assay showed that transfection of DNMT3A plasmid stimulated motility and invasion of 20(S)$\mathrm{Rg} 3$-treated cells $(200 \times)$. $t$ test. All experiments were performed in triplicate and data were showed as means $\pm \mathrm{SE}$.
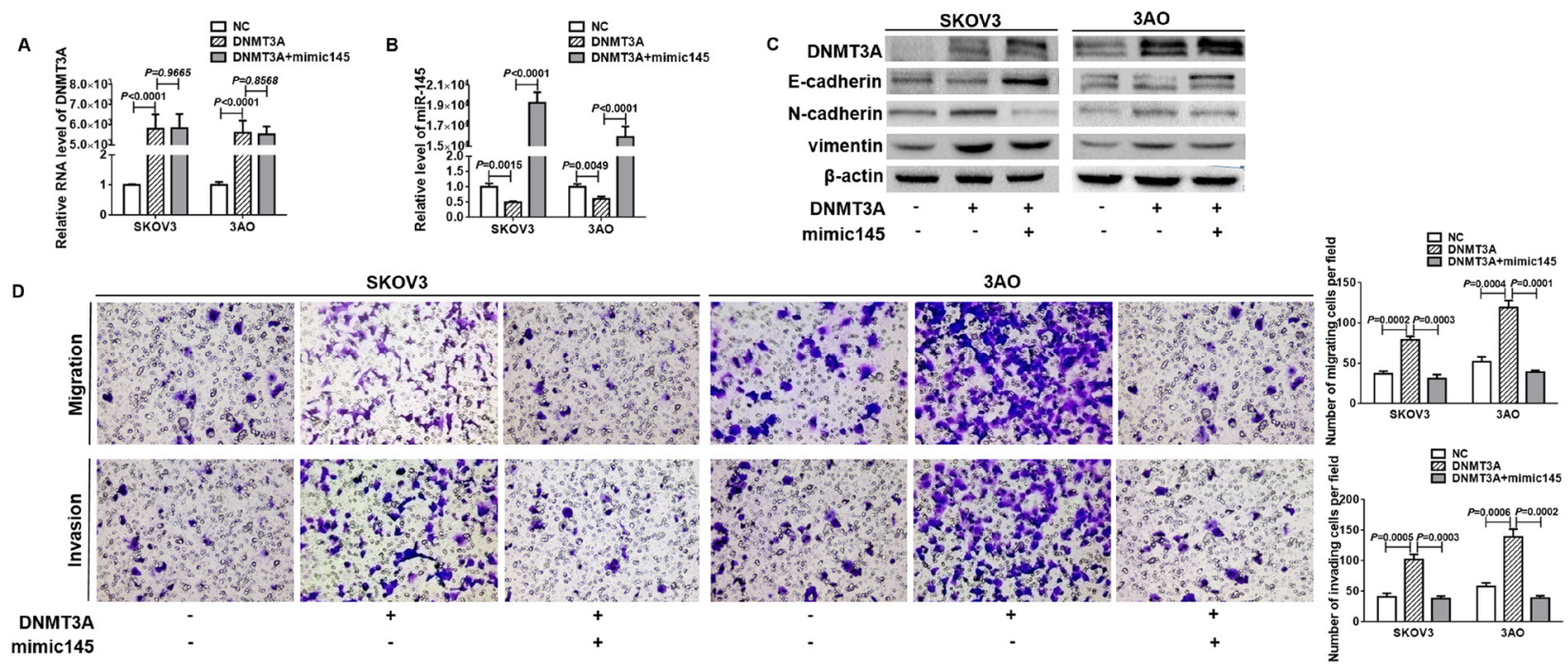

Figure 3: miR-145 blocked DNMT3A-induced EMT. (A) qRT-PCR showed that ectopic expression of miR-145 had negligible effect on DNMT3A expression in DNMT3A-overexpressed cells. (B) qRT-PCR showed that DNMT3A overexpression downregulated miR-145 expression which was recovered by mimic145 transfection. (C) Western blot analysis indicated that E-cadherin decrease, $\mathrm{N}$-cadherin and vimentin increase caused by DNMT3A were reversed by concomitant miR-145 overexpression. (D) In vitro migration and invasion assays indicated that overexpression of miR-145 largely attenuated DNMT3A promotion on the migration and invasion (200×). All experiments were performed in triplicate and datawere showed as means \pm SE. t-test. 
upregulation of E-cadherin, downregulation of N-cadherin and vimentin (Figure 4C), and attenuation of migration and invasion (Figure 4D) was largely reversed by ectopic expression of FSCN1. Meanwhile, EMT was reversed in SKOV3 and 3AO cells by downregulation of FSCN1, as shown by increased expression of E-cadherin, decreased expression of $\mathrm{N}$-cadherin and vimentin (Figure 4E), and demotion of migration and invasion (Figure 4F). Taken together, our data suggested that miR-145 blocked EMT by targeting FSCN1 in ovarian cancer cells.

\section{miR-145 inhibited ovarian cancer EMT in vivo}

To determine the effect of miR-145 on ovarian cancer progression in vivo, the primary tumor growth in nude mice were examined. miR-145-up SKOV3 cells (transfected by miR-145-expressing lentivirus) or negative control cells (NC) were subcutaneously inoculated into nude mice. All of the mice developed subcutaneous xenografts. Although no differences in body weight were detected between NC and miR-145-up groups (Figure $5 \mathrm{~A})$, the average tumor volume of miR-145-up group was reduced by nearly $40 \%$ at week 4 compared with the $\mathrm{NC}$ group (Figure 5B). Immunohistochemistry analysis of subcutaneous tumors showed that E-cadherin was higher, vimentin and FSCN1 were lower in miR-145-up tumors compared to $\mathrm{NC}$ tumors (Figure 5C).

We then examined the inhibitory effect of miR-145 on the intraperitoneal dissemination of ovarian cancer. $\mathrm{NC}$ or miR-145-up SKOV3 cells were inoculated into the abdomen of nude mice. During the 28-day observation, the average body weigh was higher in miR-145-up group than NC group, which became significant since day 22 (Figure 5D). And less metastatic tumor nodules and

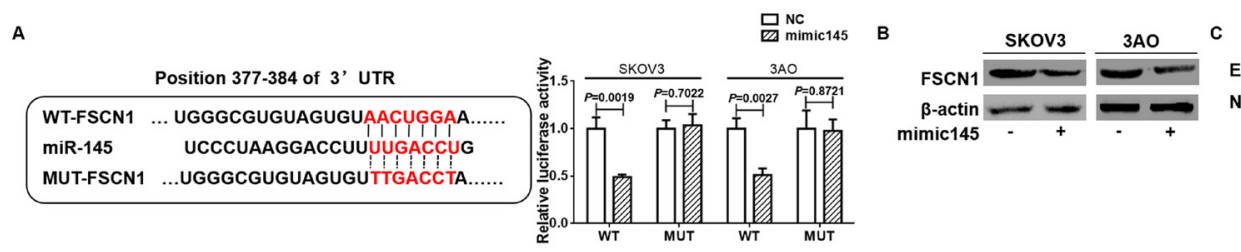

D

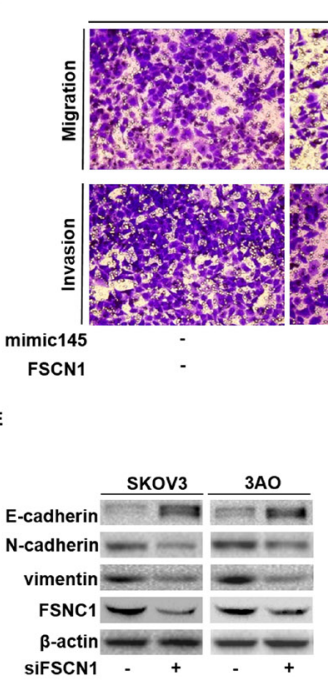

SKov3

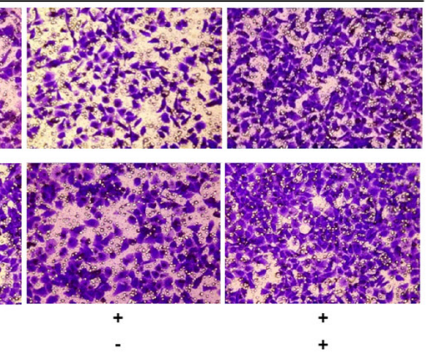

F

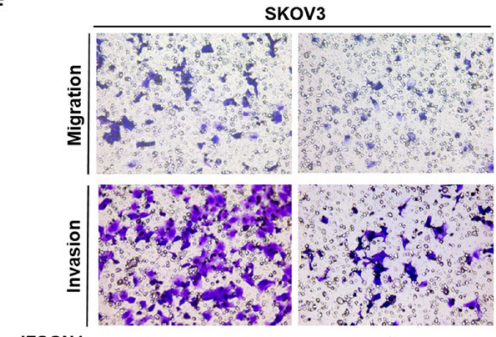

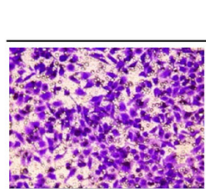

$3 A O$
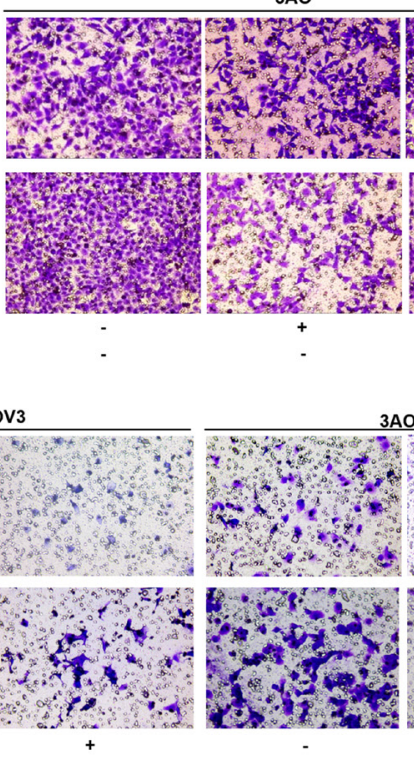
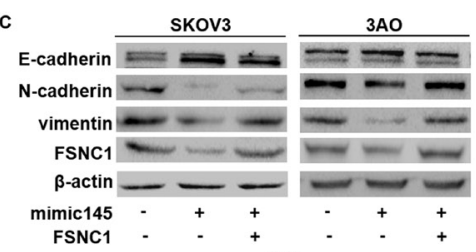

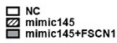

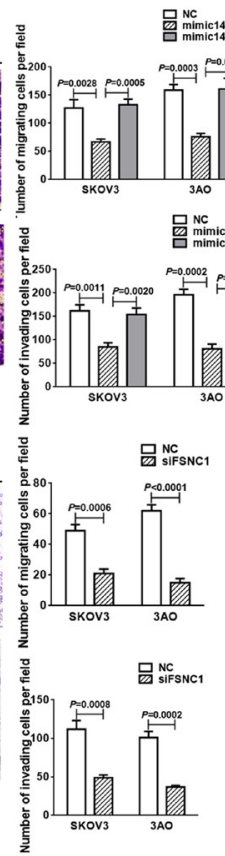

Figure 4: miR-145 directly targeted FSCNI to inhibit EMT. (A) Schematic representation of FSCN1 WT-3'UTR and MUT3'UTR sequences used for construction of luciferase report vectors. Luciferase reporter assay showed that, compared to negative control, miR-145 significantly diminished luciferase activity of wild type FSCN1 3'-UTR, while had no effect on luciferase activity of the mutated FSCN1 3'-UTR. (B) mimic145-mediated overexpression of miR-145 markedly repressed FSCN1 protein level in SKOV3 and 3AO cells. (C) Western blot assays showed that E-cadherin was deceased whereas $\mathrm{N}$-cadherin and vimentin were increased in cells by overexpression of FSCN1 and miR-145 relative to overexpression of miR-145 alone. (D) In vitro migration and invasion assays showed that FSCN1 overexpression in SKOV3 and 3AO cells largely counteracted miR-145 inhibition on cell migration and invasion $(200 \times)$. (E) Western blot assays showed that knocking down of FSCN1 caused the E-cadherin upregulation, N-cadherin and vimentin downregulation. (F) In vitro migration and invasion assays showed that FSCN1 inhibition in SKOV3 and $3 \mathrm{AO}$ cells mitigated cell motile and invading capacity $(200 \times)$. All of the treatments were carried out in triplicate, and the results are displayed as the means $\pm \mathrm{SE}$. t-test. 
ascites were developed in miR-145-up group (Figure $5 \mathrm{E})$. Intraperitoneal tumor nodules presented throughout the mice's abdominal cavity. Tumors in spleen fascia and diaphragm of miR-145-up mice were substantially smaller in size than those in $\mathrm{NC}$ mice, and immunohistochemistry analysis of metastatic tumor showed the tumors were from the intraperitoneal dissemination (Figure 5F). These data reproduced the suppressive action of miR-145 on ovarian cancer cell in vivo.

\section{DISCUSSION}

20(S)-Rg3 inhibited cancer cell motility and invasiveness, and the mechanism could be partly attributed to its anti-EMT effect [29-31]. Here, we first reported that the pathway composed of DNMT3A, miR-145, and FSCN1 was implicated in the anti-EMT mechanism of 20(S)-Rg3 (Figure 6), and that the methylation repression of miR-145 by DNMT3A played an important role in EMT occurrence.

Ginseng, is one of the oldest herbal medicines and induces a variety of physiological and pharmacological effects. Ginseng contains saponins called ginsenosides, which are considered as the biologically active ingredients in ginseng. Many researches have proposed that ginsenoside blocks EMT in cancers. Zhang and colleagues have recently shown that ginsenoside 25-OCH3-PPD, isolated from Panax notoginseng and belonged to protopanaxadiols group as 20 (S)-Rg3, reduces EMT markers in normoxically cultured breast cancer cells [32]. Xie et al. have reported that ginsenoside $\mathrm{Rg} 1$, a major active component also isolated from Panax notoginseng but belonged to protopanaxatriol group, blocks TGF $\beta 1$-induced EMT in rat renal tubular epithelial cells [33]. Collectively, these findings, together with our data, shed new light on the anti-metastasis mechanism of ginsenosides. Nevertheless, the pathway relative to the entrance of the ginsenosides into cells and the direct targets of the ginsenosides have been yet to be determined. It is worthwhile to identify the specific mechanism mediating the anti-tumor role of ginsenosides.

DNA methylation is a critical epigenetic signature that is involved in transcriptional regulation, genomic imprinting, and silencing of repetitive DNA elements [34]. Abnormal hypermethylation correlates with the transcriptional repression of multiple miRNAs. This can lead to the upregulation of oncogenic targets of microRNAs and constitutive activation of signaling pathway that can increase invasion and migration activities [35]. However, to our knowledge, there have been no
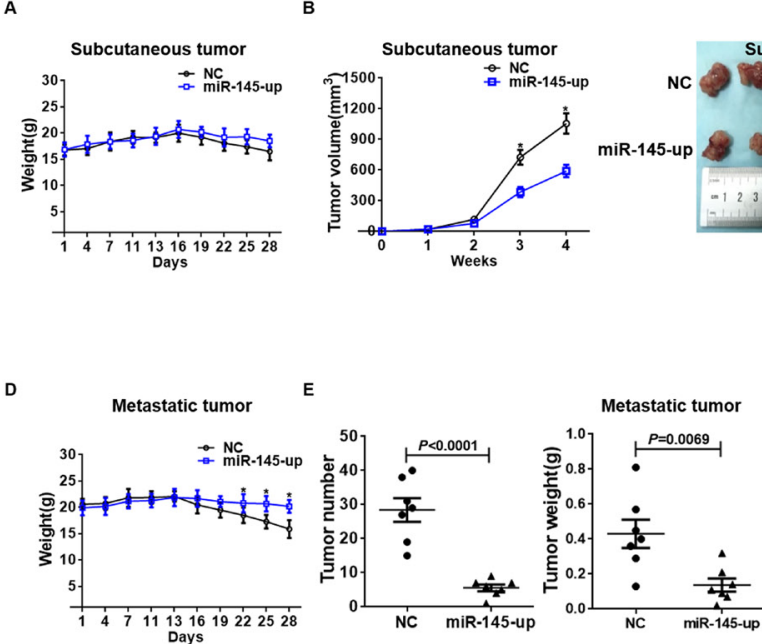

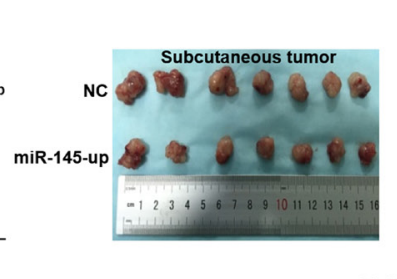

C

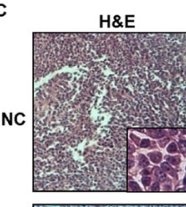

$\& \mathrm{E}$
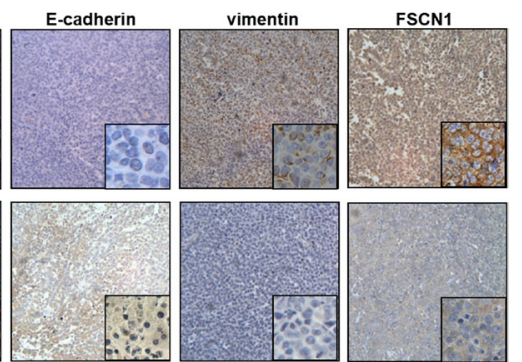

$\mathrm{F}$
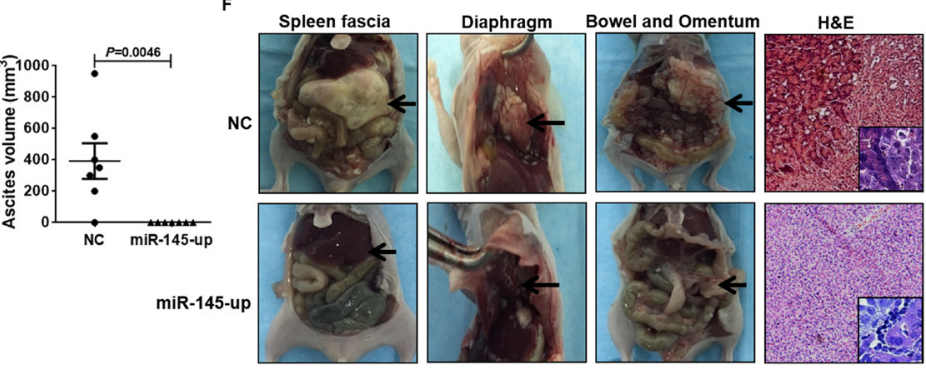

Figure 5: miR-145 inhibited ovarian cancer EMT in vivo. (A) Cells were injected subcutaneously into the nude mice flank. The body weights of nude mice with subcutaneous xenograft were monitored every other day. No differences in body weight were observed between NC and miR-145-up groups. Values are presented as the means (g) $\pm \mathrm{SD}$ ( $\mathrm{n}=7$ per groups). (B) The volumes of subcutaneous tumors were calculated for 4 weeks, which showed that miR-145 strongly inhibited tumor growth in nude mice. Data represents the mean $(\mathrm{mm} 3) \pm \mathrm{SD}$ ( $\mathrm{n}=7$ per groups). (C) Immunohistochemical staining of E-cadherin, vimentin and FSCN1 expression in subcutaneous tumor samples showed that E-cadherin was increased while vimentin and FSCN1 were decreased in miR-145 up tumors relative to that in NC group (original magnification, 100×; insets, 400×). (D) Cells were inoculated into the abdomen of nude mice. The body weights of nude mice with metastatic tumors were monitored every other day. Mice in miR-145-up group lost less body weight than NC group. (E) The overall number of metastases, tumor weight, and the volume of ascites were measured. Data are presented as the means $(\mathrm{g}) \pm \mathrm{SD}(\mathrm{n}=7 \mathrm{per}$ groups). (F) The representative images of xenografts of spleen fascia, diaphragm, bowel and omentum, and the H\&E staining of the tumor samples. The xenografts in miR-145-up group were obviously smaller in size than those in NC group. 
reports about the methylation regulation of miR-145 in ovarian cancer. In this study, we found that miR-145 was under control of DNMT3A-mediated DNA methylation, and 20(S)-Rg3 inhibited DNMT3A expression to demethylate pre-miR-145 and thus increase miR-145 expression.

DNMT3A together with DNMT3B and DNMT1 are catalytically active DNMTs responsible for genome methylation [36]. DNMT3A and DNMT3B are de novo methyltransferase with different targets [37]. DNMT1 is a maintenance DNA methyltransferase for retaining methylation pattern, with inefficient de novo methylation ability. Increasing evidence shows that these DNMTs work together to maintain a normal methylation pattern, and deregulation of either one could promote malignancies [38]. More recently, DNMT3A has been reported as contributor of EMT [39, 40]. In the present study, we showed that DNMT3A induced EMT via decreasing miR145 expression.

Decreased expression and anti-tumor function of miR-145 have been observed in several cancers [41, 42]. The mechanistic studies about miR-145 inhibition on cancer progression have initially focused on its roles in cell apoptosis, cell cycle and cell proliferation [43, 44]. Lately, miR-145 has been inversely connected to cancer cell motility and invasiveness, and the mechanism may partly be attributed to its anti-EMT effect $[45,46]$. FSCN1 [47] is a newly identified miR-145 target in a few cancers. FSCN1 is an actin-bundling protein functioning in cell movement under physiological or pathological conditions [48]. Overexpression of FSCN1 promotes migration and invasion of cancer cells [7, 49-53], and is associated with clinically unfavorable phenotypes in human epithelial cancers including EOC [48-54]. Nevertheless, the correlation of miR-145-FSCN1 with EMT has not been compellingly proven in the published data. Here we provided the evidence that overexpression of FSCN1 was sufficient to confer EMT to ovarian cancer cells, and its aberrant elevation in ovarian cancer tissues was possibly benefited from miR-145 diminution. We provided evidence that miR-145 inhibited EMT by suppressing FSCN1, however, the detailed mechanism about function of FSCN1 in EMT occurrence still necessitated further elucidation.

In conclusion, we found that $20(\mathrm{~S})-\mathrm{Rg} 3$ reversed EMT to inhibit ovarian cancer cells migration and invasion

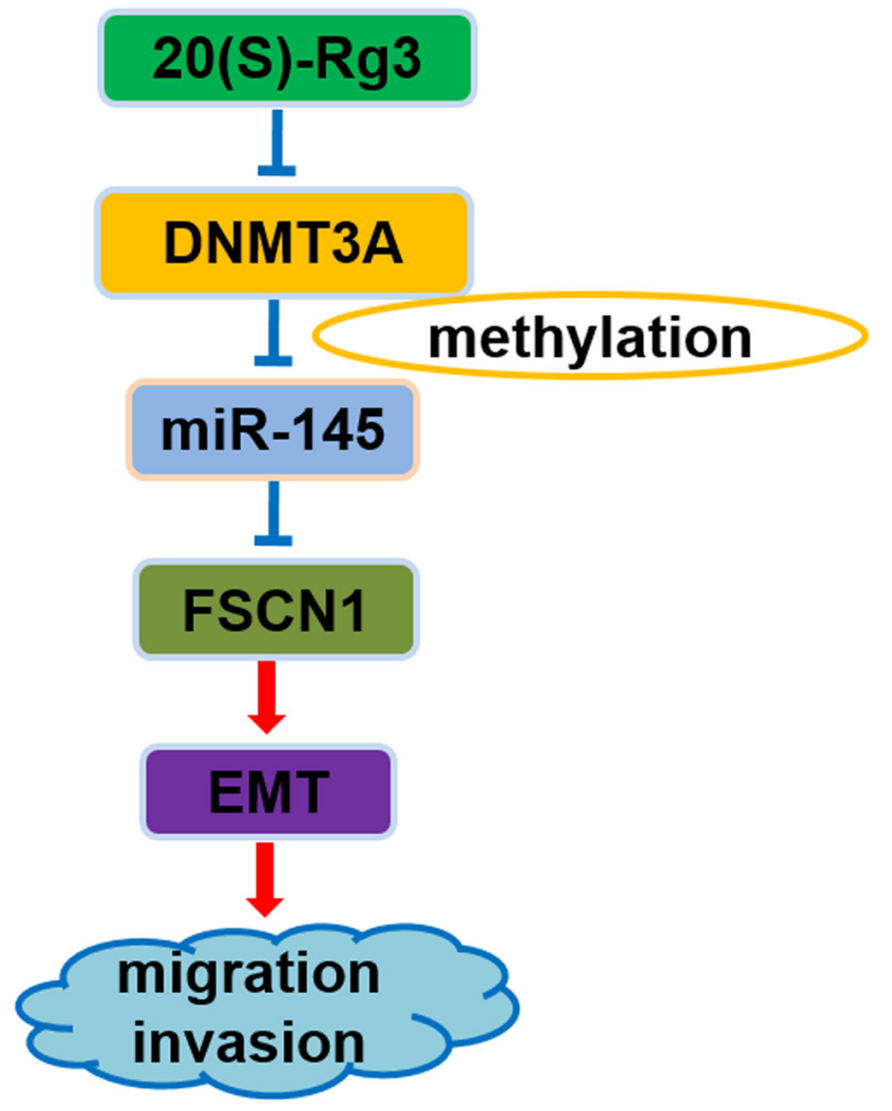

Figure 6: Schematic representation of the anti-EMT mechanism of 20(S)-Rg3. 20(S)-Rg3 down-regulated DNMT3A to demethylate pre-miR-145 and thus upregulated mature miR-145 that targeted FSCN1 and finally blocked EMT to attenuate cell migration and invasion. 
via antagonizing DNMT3A-mediated methylation of premiR-145 to promote inhibition of miR-145 on FSCN1. $20(\mathrm{~S})-\mathrm{Rg} 3$ is an efficient, low-toxicity and multi-target natural agent, and it is expected to become an important agent in the comprehensive treatment of ovarian cancer, which may improve the current strategy of ovarian cancer therapy.

\section{MATERIALS AND METHODS}

\section{Reagents and antibodies}

Ginsenoside 20(S)-Rg3 was obtained from Tasly Pharmaceutical Company (Tianjin, China) and dissolved at a concentration of $4 \mathrm{mg} / \mathrm{ml}$ in DMSO as a stock solution (stored at $-20{ }^{\circ} \mathrm{C}$ ). It was then further diluted in cell culture medium to create working concentrations. The maximum final concentration of DMSO was less than $0.1 \%$ for each treatment, and was also used as a control. Antibodies including DNMT3A, DNMT3B, E-cadherin, $\mathrm{N}$-cadherin, vimentin, and $\beta$-actin were from Cell Signaling Technology (Beverly, MA), FSCN1 was from Abcam (Cambridge, MA, USA), and DNMT1 was from Active Motif (Carlsbad, CA, USA).

\section{Cell culture and 20(S)-Rg3 treatment}

The human ovarian cancer cell line SKOV3 was obtained from the Shanghai Cell Bank of Chinese Academy of Sciences (Shanghai, China), 3AO was from the Shandong Academy of Medical Sciences (Jinan, China). Cells were maintained in RPMI 1640 medium (Gibco-BRL, Gaithersburg, MD, USA) supplemented with $10 \%(\mathrm{v} / \mathrm{v})$ fetal bovine serum at $37^{\circ} \mathrm{C}$ under a humidified $5 \% \mathrm{CO}_{2}$ atmosphere. Cells were incubated with $80 \mu \mathrm{g} /$ $\mathrm{ml}$ (for SKOV3) or $160 \mu \mathrm{g} / \mathrm{ml}$ (for $3 \mathrm{AO}$ ) of 20(S)-Rg3 for $24 \mathrm{~h}$.

\section{Quantitative real-time PCR (qRT-PCR)}

Total RNA was extracted from cells using TRIzol reagent (Invitrogen, Carlsbad, CA, USA) according to the manufacturer's instructions. Concentration and quality of total RNA were assessed by absorbance at $260 \mathrm{~nm}$ and the ratio of 260/280, respectively, on a UV spectrophotometer (BioRad Inc., Hercules, CA, USA). For mRNA detection, first-strand cDNA was synthesized using a RevertAid first strand cDNA synthesis Kit (Thermo Fisher Scientific Inc., Waltham, MA, USA). Quantitative real-time PCR was performed using a SYBR Premix Ex Taq ${ }^{\mathrm{TM}}$ II kit (Takara, Dalian, China) on a CFX96 real-time PCR system (Bio- Rad, Hercules, CA, USA). miR-145 was normalized to small nuclear U6, while DNMT3A normalized to $\beta$-actin. Relative gene expression was calculated automatically using $2^{-\Delta \Delta \mathrm{Ct}}$. Primers for miR-145 and U6 reverse transcription and amplification were designed and synthesized by
Ribo-Bio Co., Ltd. (Guangzhou, China). The following primer sequences were used: DNMT3A forward: 5'-TATTGATGAGCGCACAAGAGAGC-3'; DNMT3A reverse: 5'-GGGTGTTCCAGGGTAACATTGAG-3'; $\beta$-actin forward: 5'-TCCCTGGAGAAGAGCTACGA-3'; $\beta$-actin reverse: 5'-AGCACTGTGTTGGCGTACAG-3'.

\section{Western blot}

Total protein was collected from cells by RIPA lysis buffer containing protease inhibitors (Roche, Indianapolis, IN, USA) and $1 \mathrm{mM}$ PMSF on ice. Protein concentration was measured using the BCA200 Protein Assay kit (Pierce, Rockford, IL, USA). After heat denaturation at $100{ }^{\circ} \mathrm{C}$ for $5 \mathrm{~min}$, proteins were separated by electrophoresis on $10 \%$ SDS-PAGE gels and then transferred onto nitrocellulose membranes (Pall Life Science, NY, USA). The membranes were blocked with $5 \%$ non-fat milk at room temperature for 1 $\mathrm{h}$, and then incubated overnight at $4{ }^{\circ} \mathrm{C}$ with rabbit antihuman E-cadherin(1:1000, Cell Signaling Technology, Danvers, MA, USA), N-cadherin(1:1000, Cell Signaling Technology, Danvers, MA, USA), vimentin(1:500, Cell Signaling Technology, Danvers, MA, USA), DNMT3A (1:500, Cell Signaling Technology, Danvers, MA, USA), DNMT3B(1:500, Cell Signaling Technology, Danvers, MA, USA), DNMT1(1:1000, Active Motif; Carlsbad, CA, USA), FSCN1(1:100000, Abcam, Cambridge, MA, USA), and mouse anti-human $\beta$-actin(1:1000, Cell Signaling Technology, Danvers, MA, USA). After washing with TBST, the blots were incubated with horse radish peroxidase (HRP)-conjugated goat anti-rabbit or anti-mouse IgG. Blots were visualized using ECL reagents (Pierce, Rockford, IL, USA) by a chemiluminescence imaging system (Bio-Rad, Richmond, CA, USA).

\section{Plasmid transfection}

The human FSCN1 expression vector pLenti6/V5DEST-FASCIN was a gift from Lynda Chin (Addgene, \#31207)[55]. The human DNMT3A expression vector pcDNA3/Myc-DNMT3A was a gift from Arthur Riggs (Addgene plasmid \# 35521)[56]. SKOV3 and 3AO cells were seeded into 6-well plates until $70 \%-90 \%$ confluency and transiently transfected with pcDNA3/Myc-DNMT3A (pLenti6/V5-DEST-FASCIN) or control vector $3 \mu \mathrm{g}$ per well using the X-treme GENE HP DNA Transfection Reagent (Roche, Indianapolis, IN, USA) following the manufacturer's protocol. After 48 hours of transfection, the cells were harvested for further study.

\section{MicroRNA mimic or inhibitor transfection}

miR-145 mimic (inhibitor) and negative control were purchased from Ribo-Bio Co. Ltd. (Guangzhou, China). SKOV3 and 3AO cells were seeded into 6-well 
plates to reach $40 \%-50 \%$ confluency after $24 \mathrm{~h}$ and then transiently transfected with $60 \mathrm{nM}$ miR-145 mimic (SKOV3) or $100 \mathrm{nM}$ miR-145 inhibitor or negative control using the X-treme GENE siRNA Transfection Reagent (Roche, Indianapolis, IN, USA). After $24 \mathrm{~h}$ of transfection, the cells were treated with $80 \mu \mathrm{g} / \mathrm{ml}(\mathrm{SKOV} 3)$ and $160 \mu \mathrm{g} /$ $\mathrm{ml}(3 \mathrm{AO})$ of $20(\mathrm{~S})-\mathrm{Rg} 3$ for $24 \mathrm{~h}$.

\section{siRNA and transient transfection}

Human FSCN1 siRNA was purchased from GenePharma (Shanghai, China). Ovarian cancer cells were seeded into 6-well plates until they reached $40 \%-50 \quad \%$ confluency. FSCN1 siRNA (GCAGCCTGAAGAAGAAGCA) was transiently transfected 100nM per well using the X-treme GENE siRNA Transfection Reagent (Roche, Indianapolis, IN, USA). After 48 hours transfection, the cells were harvested for further studies.

\section{Cell migration and invasion assay}

After treated, cells were trypsinized and counted. A total of $1 \times 10^{5}$ cells (for migration assay) or $5 \times 10^{5}$ cells (for invasion assay) in $100 \mu \mathrm{l}$ serum-free medium were added into millicells (Millipore Co., Bedford, MA, USA) without (for migration assay) or with (for invasion assay) Matrigel (Becton Dickinson Labware, Bedford, MA, USA) coated. $500 \mu \mathrm{l}$ of 1640 medium containing $20 \%$ newborn bovine serum was added to the bottom chambers as the chemotactic factor. After incubation for $24 \mathrm{~h}$ (for migration assay) or $48 \mathrm{~h}$ (for invasion assay) at $37^{\circ} \mathrm{C}$, cells remaining on the upper surface of the filter were removed using cotton swabs. Then the migrated cells were fixed using methyl alcohol and stained using $0.1 \%$ crystal violet. Migratory (or invasive) cells were counted and averaged from images of five random fields (original magnification $\times 200$ ) captured using an inverted light microscope. The mean values of three duplicate assays were used for statistical analysis.

\section{DNA bisulfite modification and methylation- specific PCR (MSP)}

Cells treated by $80 \mu \mathrm{g} / \mathrm{ml}$ (SKOV3) and $160 \mu \mathrm{g} /$ $\mathrm{ml}(3 \mathrm{AO})$ for $24 \mathrm{~h}$ in 24-well plates were trypsinized and resuspended in cold PBS at the concentration of $\sim 6 \times 10^{6} / \mathrm{ml}$. DNA bisulfite modification and purification were performed using an EZ DNA methylation-Direct kit (ZYMO RESEARCH, California, USA) according to its instructions. Concentration of DNA was evaluated by absorbance at $260 \mathrm{~nm}$ on a UV spectrophotometer (BioRad Inc., Hercules, CA, USA). The set of primers for MSP was flanking the $3 \mathrm{~kb} 5$ '-region upstream from the start of premiR-145 sequence. The primers for methylation-specific PCR were designed by MethPrimer and the sequences were as follows: methylated (M) forward: 5'-GGAGATT
GGGGAATATATATGAGTC-3'; methylated (M) reverse: 5'-AAAATAAAATACCACACGTCGC-3'; unmethylated (U)- forward: 5'-AGATTGGGGAATATATATGAGTT GT-3'; unmethylated (U)- reverse: 5'-ACCAAAATA AAATACCACACATCAC-3'. DNA amplification was performed with Epi Taq HS (Takara, Dalian, China) under the following condition: $94^{\circ} \mathrm{C}$ for $5 \mathrm{~min} ; 30$ cycles of 94 ${ }^{\circ} \mathrm{C}$ for $30 \mathrm{~s}, 50{ }^{\circ} \mathrm{C}$ for $30 \mathrm{~s}, 72{ }^{\circ} \mathrm{C}$ for $30 \mathrm{~s}$; and $72{ }^{\circ} \mathrm{C}$ for $10 \mathrm{~min}$. The PCR productions were separated by $2.0 \%$ agarose gel electrophoresis and visualized by a Bio-Rad image lab system (Richmond, CA, USA).

\section{Luciferase reporter plasmid construction}

The wild-type 3'-UTR sequence of the target gene carrying a putative miR-145 binding site was amplified by PCR. To generate mutant 3'-UTR fragment of miR145 target gene, we adopted the two-step PCR method as reported previously. Briefly, two fragments of 3'UTR were amplified by two sets of overlapped primers in which mutated seeding sequences of miR-145 were introduced. The wild-type and mutant PCR products were digested with Hind III and Sac I enzymes, inserted into pMIR-REPORT ${ }^{\mathrm{TM}}$ Luciferase vectors (Ambion, Austin, TX, USA) and verified by DNA sequencing. The following primers were used: FSCN1-WT 3'UTR forward: 5 '-CCTCGCTCTGGGAGTACTAGGG-3'; FSCN1-WT 3'UTR reverse: 5' -CTGGGGCTGCAGACTGAGTTAT-3'; FSCN1-MUT 3'UTR P1: 5'-GCGGCTCGAGCCTCGCTCTGGGAGT ACTAGGG-3'; P2: 5'-AATGCGGCCGCCTGGGGCTG CAGACTGAGTTAT-3'; P3: 5'-CAAAAGATAGGTCAA ACACTACACGCCCAGGGC-3'; P4: 5'-TGTAGTGTTT GACCTATCTTTTGCCTCTCC CAG-3'.

\section{Luciferase reporter assay}

Luciferase reporter assays were carried out in both SKOV3 and $3 \mathrm{AO}$ cells. Cells were seeded into 24-well plate. When reached $80 \%-90 \%$ confluency, cells were co-transfected with pRL-TK vector (20 ng), wild-type (WT-3' UTR) or mutant (MUT-3' UTR) reporter vectors (180 ng), along with miR-145 mimic or negative control at a final concentration of $20 \mathrm{nM}$ using the X-treme GENE siRNA Transfection Reagent. Transfections were performed in triplicate. $24 \mathrm{~h}$ after transfection, the relative firefly luciferase activity (normalized to Renilla luciferase activity) was measured using a dual-luciferase reporter gene assay system (Promega, Madison, WI, USA), and results were depicted as the percentage change over the respective control. Each experiment was performed in triplicate.

\section{Animal study}

The care and use of experimental animals were approved by the ethical committee of the First 
Affiliated Hospital of Xi'an Jiaotong University, and were adherent to the institutional guidelines and ethical standards. SKOV3 cells were infected with miR-145expressing lentivirus GV209-miR145 (GENECHEM, Shanghai, China) or negative control lentivirus GV209 (GENECHEM, Shanghai, China). For subcutaneous xenograft experiment, cells were trypsinized and resuspended in PBS at a final concentration of $2 \times 10^{7}$ cells/ ml. $2 \times 10^{6}$ cells were injected subcutaneously into the flank of six-week old BALB/c nude mice. Animal body weights and tumor perpendicular diameters were recorded every two days. Tumor volumes were calculated according to the formula of $\mathrm{V}=0.5236 \times\left(\mathrm{L} \times \mathrm{W}^{2}\right)(\mathrm{V}$ : tumor volume, L: length, W: width). The mice were killed after 28 days on the experimental treatments. Tumor samples were fixed in $4 \%$ polyformaldehyde for $24 \mathrm{~h}$ at room temperature, paraffin-embedded, and sectioned for immunohistochemical analysis. For intraperitoneal xenograft model, SKOV3 cells were trypsinized and resuspended in PBS at a concentration of $1 \times 10^{8} / \mathrm{ml}$. Mice were inoculated with $1 \times 10^{7}$ cells in the abdominal cavity at day 0 . The mice were observed daily and sacrificed after 28 days. Necropsies were performed, disseminated lesions were resected from mice and pathologically confirmed using HE staining. Ascitic fluid was collected by $1 \mathrm{~mL}$ syringe and measured by pipet. The number and weight of tumor nodules on the surface of the spleen fascia, diaphragm, bowel and omentum, liver and bladder were counted, measured and statistically analyzed.

\section{Immunohistochemistry analysis}

Paraffin-embedded xenograft tissue sections on poly-1-lysine-coated slides were deparaffinized and rinsed with $10 \mathrm{mM}$ Tris- $\mathrm{HCl}$ (pH 7.4) and $150 \mathrm{mM}$ sodium chloride. Paroxidase was quenched with methanol and 3 $\%$ hydrogen peroxide. Slides were then placed in $10 \mathrm{mM}$ citrate buffer $(\mathrm{pH} 6.0)$ at $100^{\circ} \mathrm{C}$ for $20 \mathrm{~min}$ in a pressurized heating chamber. Detection of antigens was carried out using incubation with the primary antibodies for 2 hours at room temperature, followed by incubation with HRPlabeled secondary antibody (MaxVision ${ }^{\mathrm{TM}}$ HRP-Polymer anti-Mouse/Rabbit IHC Kit) at room temperature for 30 minutes and color development with DAB. Negative control specimens were incubated in PBS without the primary antibody under the same conditions. Slides were counterstained with hematoxylin, dehydrated in an ascending alcohol series, and mounted for analysis. Digital images were acquired on an Olympus BH-2 microscope (Tokyo, Japan) installed with the DeltaPix Camera and software (Maalov, Denmark).

\section{Statistical analysis}

All experiments were performed at least in triplicate, and each experiment was independently performed at least 3 times. The graphical presentations were performed using GraphPad Prism 5.0. Data were presented as the means \pm SE and were analyzed using SPSS 22.0 software (Chicago, IL, USA). Statistical differences were tested by Chi-square test or two-tailed t-test, and Fisher exact test was performed to analysis MSP results. Differences were considered significant at $\mathrm{P}<0.05\left(^{*}\right)$ or highly significant at $\mathrm{P}<0.001(* *)$.

\section{ACKNOWLEDGMENTS}

This work was supported by the National Natural Science Foundation of China: No.30973429.

\section{CONFLICTS OF INTEREST}

The authors have no conflicts of interest to disclose.

\section{REFERENCES}

1. Siegel R, Ma J, Zou Z, Jemal A. Cancer statistics, 2014. CA Cancer J Clin. 2014; 64:9-29.

2. Elzek MA, Rodland KD. Proteomics of ovarian cancer: functional insights and clinical applications. Cancer Metastasis Rev. 2015; 34:83-96.

3. Huang RY, Chung VY, Thiery JP. Targeting pathways contributing to epithelial-mesenchymal transition (EMT) in epithelial ovarian cancer. Curr Drug Targets. 2012; 13:1649-1653.

4. Gillis CN. Panax ginseng pharmacology: a nitric oxide link? Biochem Pharmacol. 1997; 54:1-8.

5. Liao B, Newmark H, Zhou R. Neuroprotective effects of ginseng total saponin and ginsenosides $\mathrm{Rb} 1$ and $\mathrm{Rg} 1$ on spinal cord neurons in vitro. Exp Neurol. 2002; 173:224-234.

6. Nag SA, Qin JJ, Wang W, Wang MH, Wang H, Zhang R. Ginsenosides as anticancer agents: in vitro and in vivo activities, structure-activity relationships, and molecular mechanisms of action. Front Pharmacol. 2012; 3:25.

7. Lu JM, Yao Q, Chen C. Ginseng compounds: an update on their molecular mechanisms and medical applications. Curr Vasc Pharmacol. 2009; 7:293-302.

8. Lee SY, Kim GT, Roh SH, Song JS, Kim HJ, Hong SS, Kwon SW, Park JH. Proteomic analysis of the anti-cancer effect of 20S-ginsenoside Rg3 in human colon cancer cell lines. Biosci Biotechnol Biochem. 2009; 73:811-816.

9. Mochizuki M, Yoo YC, Matsuzawa K, Sato K, Saiki I, Tono-Oka S, Samukawa K, Azuma I. Inhibitory effect of tumor metastasis in mice by saponins, ginsenoside- $\mathrm{Rb}$, 20(R)- and 20(S)-ginsenoside-Rg3, of red ginseng. Biol Pharm Bull. 1995; 18:1197-1202.

10. Bartel DP. MicroRNAs: genomics, biogenesis, mechanism, and function. Cell. 2004; 116:281-297.

11. Tan J, Qiu K, Li M, Liang Y. Double-negative feedback loop between long non-coding RNA TUG1 and 
miR-145 promotes epithelial to mesenchymal transition and radioresistance in human bladder cancer cells. FEBS Lett. 2015; 589:3175-3181.

12. Ju BL, Chen YB, Zhang WY, Yu CH, Zhu DQ, Jin J. miR145 regulates chemoresistance in hepatocellular carcinoma via epithelial mesenchymal transition. Cell Mol Biol (Noisy-le-grand). 2015; 61:12-16.

13. Somarelli JA, Shelter S, Jolly MK, Wang X, Bartholf Dewitt S, Hish AJ, Gilja S, Eward WC, Ware KE, Levine H, Armstrong AJ, Garcia-Blanco MA. Mesenchymal-epithelial transition in sarcomas is controlled by the combinatorial expression of miR-200s and GRHL2. Mol Cell Biol. 2016; 36:2503-2513.

14. Teng Y, Zhao L, Zhang Y, Chen W, Li X. Id-1, a protein repressed by miR-29b, facilitates the TGFbeta1-induced epithelial-mesenchymal transition in human ovarian cancer cells. Cell Physiol Biochem. 2014; 33:717-730.

15. Chan M, Liaw CS, Ji SM, Tan HH, Wong CY, Thike AA, Tan PH, Ho GH, Lee AS. Identification of circulating microRNA signatures for breast cancer detection. Clin Cancer Res. 2013; 19:4477-4487.

16. Xu Q, Liu LZ, Qian X, Chen Q, Jiang Y, Li D, Lai L, Jiang BH. MiR-145 directly targets p70S6K1 in cancer cells to inhibit tumor growth and angiogenesis. Nucleic Acids Res. 2012; 40:761-774.

17. Shi B, Sepp-Lorenzino L, Prisco M, Linsley P, deAngelis T, Baserga R. Micro RNA 145 targets the insulin receptor substrate-1 and inhibits the growth of colon cancer cells. J Biol Chem. 2007; 282:32582-32590.

18. Cho WC, Chow AS, Au JS. MiR-145 inhibits cell proliferation of human lung adenocarcinoma by targeting EGFR and NUDT1. RNA Biol. 2011; 8:125-131.

19. Gramantieri L, Fornari F, Ferracin M, Veronese A, Sabbioni S, Calin GA, Grazi GL, Croce CM, Bolondi L, Negrini M. MicroRNA-221 targets Bmf in hepatocellular carcinoma and correlates with tumor multifocality. Clin Cancer Res. 2009; 15:5073-5081.

20. Zhang J, Guo H, Zhang H, Wang H, Qian G, Fan X, Hoffman AR, Hu JF, Ge S. Putative tumor suppressor miR145 inhibits colon cancer cell growth by targeting oncogene Friend leukemia virus integration 1 gene. Cancer. 2011; 117:86-95

21. Sachdeva M, Zhu S, Wu F, Wu H, Walia V, Kumar S, Elble R, Watabe K, Mo YY. p53 represses c-Myc through induction of the tumor suppressor miR-145. Proc Natl Acad Sci U S A. 2009; 106:3207-3212.

22. Zhang J, Guo H, Qian G, Ge S, Ji H, Hu X, Chen W. MiR145 , a new regulator of the DNA fragmentation factor-45 (DFF45)-mediated apoptotic network. Mol Cancer. 2010; 9:211.

23. Sachdeva M, Mo YY. MicroRNA-145 suppresses cell invasion and metastasis by directly targeting mucin 1 . Cancer Res. 2010; 70:378-387.
24. Lopez-Serra P, Esteller M. DNA methylation-associated silencing of tumor-suppressor microRNAs in cancer. Oncogene. 2012; 31:1609-1622.

25. Gao Q, Steine EJ, Barrasa MI, Hockemeyer D, Pawlak M, Fu D, Reddy S, Bell GW, Jaenisch R. Deletion of the de novo DNA methyltransferase Dnmt3a promotes lung tumor progression. Proc Natl Acad Sci U S A. 2011; 108:18061-18066.

26. Bai X, Song Z, Fu Y, Yu Z, Zhao L, Zhao H, Yao W, Huang D, Mi X, Wang E, Zheng Z, Wei M. Clinicopathological significance and prognostic value of DNA methyltransferase $1,3 \mathrm{a}$, and $3 \mathrm{~b}$ expressions in sporadic epithelial ovarian cancer. PloS One. 2012; 7:e40024.

27. Suh SO, Chen Y, Zaman MS, Hirata H, Yamamura S, Shahryari V, Liu J, Tabatabai ZL, Kakar S, Deng G, Tanaka Y, Dahiya R. MicroRNA-145 is regulated by DNA methylation and p53 gene mutation in prostate cancer. Carcinogenesis. 2011; 32:772-778.

28. Kano M, Seki N, Kikkawa N, Fujimura L, Hoshino I, Akutsu Y, Chiyomaru T, Enokida H, Nakagawa M, Matsubara H. miR-145, miR-133a and miR-133b: tumorsuppressive miRNAs target FSCN1 in esophageal squamous cell carcinoma. Int J Cancer. 2010; 127:2804-2814.

29. Tian L, Shen D, Li X, Shan X, Wang X, Yan Q, Liu J. Ginsenoside Rg3 inhibits epithelial-mesenchymal transition (EMT) and invasion of lung cancer by down-regulating FUT4. Oncotarget. 2016; 7:1619-1632. doi: 10.18632/ oncotarget.6451.

30. Kim YJ, Choi WI, Jeon BN, Choi KC, Kim K, Kim TJ, Ham J, Jang HJ, Kang KS, Ko H. Stereospecific effects of ginsenoside 20-Rg3 inhibits TGF-beta1-induced epithelialmesenchymal transition and suppresses lung cancer migration, invasion and anoikis resistance. Toxicology. 2014; 322:23-33.

31. Liu T, Zhao L, Zhang Y, Chen W, Liu D, Hou H, Ding L, Li X. Ginsenoside 20(S)-Rg3 targets HIF-1alpha to block hypoxia-induced epithelial-mesenchymal transition in ovarian cancer cells. PloS One. 2014; 9:e103887.

32. Wang W, Zhang X, Qin JJ, Voruganti S, Nag SA, Wang MH, Wang H, Zhang R. Natural product ginsenoside 25-OCH3PPD inhibits breast cancer growth and metastasis through down-regulating MDM2. PloS One. 2012; 7:e41586.

33. Xie XS, Yang M, Liu HC, Zuo C, Li HJ, Fan JM. Ginsenoside $\mathrm{Rg} 1$, a major active component isolated from Panax notoginseng, restrains tubular epithelial to myofibroblast transition in vitro. J Ethnopharmacol. 2009; 122:35-41.

34. Bartel DP. MicroRNAs: target recognition and regulatory functions. Cell. 2009; 136:215-233.

35. Duraisamy S, Kufe T, Ramasamy S, Kufe D. Evolution of the human MUC1 oncoprotein. Int J Oncol. 2007; 31:671-677.

36. Robertson $\mathrm{KD}$, Uzvolgyi E, Liang G, Talmadge C, Sumegi J, Gonzales FA, Jones PA. The human DNA methyltransferases (DNMTs) 1, 3a and 3b: coordinate 
mRNA expression in normal tissues and overexpression in tumors. Nucleic Acids Res. 1999; 27:2291-2298.

37. Okano M, Bell DW, Haber DA, Li E. DNA methyltransferases Dnmt3a and Dnmt3b are essential for de novo methylation and mammalian development. Cell. 1999; 99:247-257.

38. Liang G, Chan MF, Tomigahara Y, Tsai YC, Gonzales FA, Li E, Laird PW, Jones PA. Cooperativity between DNA methyltransferases in the maintenance methylation of repetitive elements. Mol Cell Biol. 2002; 22:480-491.

39. Liu F, Zhou Y, Zhou D, Kan M, Niu X, Zhang Z, Zhang D, Tao L, He L, Zhan L, Liu Y. Whole DNA methylome profiling in lung cancer cells before and after epithelial-tomesenchymal transition. Diagn Pathol. 2014; 9:66.

40. Bu F, Liu X, Li J, Chen S, Tong X, Ma C, Mao H, Pan F, Li X, Chen B, Xu L, Li E, Kou G, et al. TGF-beta1 induces epigenetic silence of TIP30 to promote tumor metastasis in esophageal carcinoma. Oncotarget. 2015; 6:2120-2133. doi: 10.18632/oncotarget.2940.

41. Khan S, Ebeling MC, Zaman MS, Sikander M, Yallapu MM, Chauhan N, Yacoubian AM, Behrman SW, Zafar N, Kumar D, Thompson PA, Jaggi M, Chauhan SC. MicroRNA-145 targets MUC13 and suppresses growth and invasion of pancreatic cancer. Oncotarget. 2014; 5:75997609. doi: 10.18632/oncotarget.2281.

42. Dong R, Liu X, Zhang Q, Jiang Z, Li Y, Wei Y, Yang Q, Liu J, Wei JJ, Shao C, Liu Z, Kong B. miR-145 inhibits tumor growth and metastasis by targeting metadherin in high-grade serous ovarian carcinoma. Oncotarget. 2014; 5:10816-10829. doi: 10.18632/oncotarget.2522.

43. Arndt GM, Dossey L, Cullen LM, Lai A, Druker R, Eisbacher M, Zhang C, Tran N, Fan H, Retzlaff K, Bittner A, Raponi M. Characterization of global microRNA expression reveals oncogenic potential of miR-145 in metastatic colorectal cancer. BMC Cancer. 2009; 9:374.

44. Iorio MV, Ferracin M, Liu CG, Veronese A, Spizzo R, Sabbioni S, Magri E, Pedriali M, Fabbri M, Campiglio M, Menard S, Palazzo JP, Rosenberg A, et al. MicroRNA gene expression deregulation in human breast cancer. Cancer Res. 2005; 65:7065-7070.

45. Hu J, Qiu M, Jiang F, Zhang S, Yang X, Wang J, Xu L, Yin R. MiR-145 regulates cancer stem-like properties and epithelial-to-mesenchymal transition in lung adenocarcinoma-initiating cells. Tumour Biol. 2014; 35:8953-8961.

46. Lu R, Ji Z, Li X, Zhai Q, Zhao C, Jiang Z, Zhang S, Nie L, Yu Z. miR-145 functions as tumor suppressor and targets two oncogenes, ANGPT2 and NEDD9, in renal cell carcinoma. J Cancer Res Clin Oncol. 2014; 140:387-397.

47. Zhang Y, Lin Q. MicroRNA-145 inhibits migration and invasion by down-regulating FSCN1 in lung cancer. Int $\mathrm{J}$ Clin Exp Med. 2015; 8:8794-8802.

48. Hashimoto Y, Skacel M, Adams JC. Roles of fascin in human carcinoma motility and signaling: prospects for a novel biomarker? Int J Biochem Cell Biol. 2005; 37:1787-1804.

49. Jawhari AU, Buda A, Jenkins M, Shehzad K, Sarraf C, Noda M, Farthing MJ, Pignatelli M, Adams JC. Fascin, an actin-bundling protein, modulates colonic epithelial cell invasiveness and differentiation in vitro. Am J Pathol. 2003; 162:69-80.

50. Hashimoto Y, Parsons M, Adams JC. Dual actin-bundling and protein kinase $\mathrm{C}$-binding activities of fascin regulate carcinoma cell migration downstream of Rac and contribute to metastasis. Mol Biol Cell. 2007; 18:4591-4602.

51. Darnel AD, Behmoaram E, Vollmer RT, Corcos J, Bijian K, Sircar K, Su J, Jiao J, Alaoui-Jamali MA, Bismar TA. Fascin regulates prostate cancer cell invasion and is associated with metastasis and biochemical failure in prostate cancer. Clin Cancer Res. 2009; 15:1376-1383.

52. Hashimoto $\mathrm{Y}$, Ito $\mathrm{T}$, Inoue $\mathrm{H}$, Okumura $\mathrm{T}$, Tanaka $\mathrm{E}$, Tsunoda S, Higashiyama M, Watanabe G, Imamura M, Shimada Y. Prognostic significance of fascin overexpression in human esophageal squamous cell carcinoma. Clin Cancer Res. 2005; 11:2597-2605.

53. Xie JJ, Xu LY, Zhang HH, Cai WJ, Mai RQ, Xie YM, Yang ZM, Niu YD, Shen ZY, Li EM. Role of fascin in the proliferation and invasiveness of esophageal carcinoma cells. Biochem Biophys Res Commun. 2005; 337:355-362.

54. Hayashi Y, Osanai M, Lee GH. Fascin-1 expression correlates with repression of E-cadherin expression in hepatocellular carcinoma cells and augments their invasiveness in combination with matrix metalloproteinases. Cancer Sci. 2011; 102:1228-1235.

55. Scott KL, Nogueira C, Heffernan TP, van Doorn R, Dhakal S, Hanna JA, Min C, Jaskelioff M, Xiao Y, Wu CJ, Cameron LA, Perry SR, Zeid R, et al. Proinvasion metastasis drivers in early-stage melanoma are oncogenes. Cancer Cell. 2011; 20:92-103.

56. Chen ZX, Mann JR, Hsieh CL, Riggs AD, Chedin F. Physical and functional interactions between the human DNMT3L protein and members of the de novo methyltransferase family. J Cell Biochem. 2005; 95:902-917. 